\title{
Anti-prion Drugs Targeting the Protein Folding Activity of the Ribosome Reduce PABPN1 Aggregation
}

\author{
Aline Bamia $^{1} \cdot$ Maha Sinane $^{1} \cdot$ Rima Naït-Saïdi $^{2} \cdot$ Jamila Dhiab $^{3} \cdot$ Marc Keruzoré $^{1} \cdot$ Phu Hai Nguyen ${ }^{1,4}$.

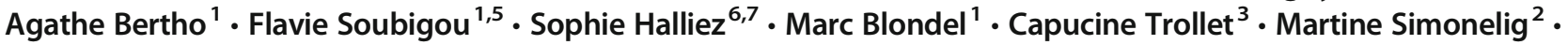 \\ Gaëlle Friocourt ${ }^{1}$ - Vincent Béringue ${ }^{6} \cdot$ Frédéric Bihel $^{8} \cdot$ Cécile Voisset $^{1}$ (I)
}

Accepted: 13 December 2020 / Published online: 2 February 2021

(C) The American Society for Experimental NeuroTherapeutics, Inc. 2021

\begin{abstract}
Prion diseases are caused by the propagation of $\mathrm{PrP}^{\mathrm{Sc}}$, the pathological conformation of the $\operatorname{PrP}^{\mathrm{C}}$ prion protein. The molecular mechanisms underlying $\mathrm{PrP}^{\mathrm{Sc}}$ propagation are still unsolved and no therapeutic solution is currently available. We thus sought to identify new anti-prion molecules and found that flunarizine inhibited $\mathrm{PrP}^{\mathrm{Sc}}$ propagation in cell culture and significantly prolonged survival of prion-infected mice. Using an in silico therapeutic repositioning approach based on similarities with flunarizine chemical structure, we tested azelastine, duloxetine, ebastine, loperamide and metixene and showed that they all have an anti-prion activity. Like flunarizine, these marketed drugs reduced $\mathrm{PrP}^{\mathrm{Sc}}$ propagation in cell culture and in mouse cerebellum organotypic slice culture, and inhibited the protein folding activity of the ribosome (PFAR). Strikingly, some of these drugs were also able to alleviate phenotypes due to PABPN1 nuclear aggregation in cell and Drosophila models of oculopharyngeal muscular dystrophy (OPMD). These data emphasize the therapeutic potential of anti-PFAR drugs for neurodegenerative and neuromuscular proteinopathies.
\end{abstract}

Key Words Prions $\cdot \mathrm{PrP}^{\mathrm{Sc}} \cdot$ drug repositioning $\cdot \mathrm{PFAR} \cdot \mathrm{OPMD} \cdot \mathrm{PABPN} 1$

\section{Introduction}

Although the concept of infectious proteins was first established for the prion protein PrP in mammals suffering from transmissible spongiform encephalopathy [1], prions are broadly found in other model organisms including the budding yeast $S$. cerevisiae. Over the last years, we have taken advantage of the existence of prions in yeast to identify new anti-prion compounds by screening drugs able to cure $[\mathrm{PSI}+]$ and [URE3] yeast prions [2-7]. Some of the compounds we

Aline Bamia and Maha Sinane contributed equally to this work, Rima Naït-Saïdi and Jamila Dhiab contributed equally to this work, Frédéric Bihel and Cécile Voisset contributed equally to this work.

Frédéric Bihel

frederic.bihel@unistra.fr

Cécile Voisset

cecile.voisset@univ-brest.fr

Inserm, Univ Brest, EFS, UMR 1078, GGB, F-29200 Brest, France

2 Institute of Human Genetics, UMR9002 CNRS-Univ Montpellier, mRNA Regulation and Development, Montpellier, France

3 Sorbanne Université, Inserm, Institut de Myologie, Centre de Recherche en Myologie, F75013 Paris, France

4 Present address: Host Parasite Interactions Section, Laboratory of Intracellular Parasites, NIAID, NIH, Rocky Mountain Laboratories, Hamilton, MT, USA
5 Present address: Centre for Gene Regulation and Expression, Sir James Black Centre, School of Life Sciences, University of Dundee, Dundee DD1 5EH, UK

6 INRAE, UVSQ, VIM, Université Paris-Saclay, Jouy-en-Josas, France

7 Inserm, CHU Lille, U1172 - LilNCog - Lille Neuroscience \& Cognition, Univ. Lille, F-59000 Lille, France

Laboratoire d'Innovation Thérapeutique, LIT, UMR7200, IMS MEDALIS, Faculty of Pharmacy, CNRS, Université de Strasbourg, Illkirch F-67400, France 
have identified are active both in vitro and in vivo against $\operatorname{PrP}^{\mathrm{Sc}}$ mammalian prions, demonstrating that some of the molecular mechanisms controlling prion onset and propagation are similar in yeast and mammals $[2-6,8,9]$. In particular, we identified two FDA-approved drugs, guanabenz (GA) and imiquimod (IQ), and 6-aminophenanthridine (6AP), with antiprion activity $[4-6,9,10]$. A reverse screening approach has allowed us to identify one of the cellular targets of these compounds: the domain $\mathrm{V}$ of the large ribosomal RNA (rRNA) of the large 60S ribosome subunit [4-6, 9, 10]. The domain V of large rRNA (23S in E. coli, $25 \mathrm{~S}$ in S. cerevisiae, $28 \mathrm{~S}$ in metazoans) is a ribozyme carrying 2 enzymatic activities: (i) the peptidyl transferase activity and (ii) the protein folding activity, PFAR (protein folding activity of the ribosome), which can refold denatured proteins back to their functionally active forms $[11,12]$. Ribosomal RNA from all three kingdoms of life exhibit a protein folding activity [12], which is in good agreement with the fact that both sequence and secondary structure of rRNA domain $\mathrm{V}$ are highly conserved [13]. 6AP, GA and IQ were shown to be the first described competitive inhibitors of PFAR that do not affect protein translation $[5,6,14]$. The fact that these three anti-prion compounds are also PFAR inhibitors led us to explore the link between PFAR and prion propagation. We found that PFAR is involved in the de novo formation and propagation of [PSI+] prion in yeast [15], strongly suggesting that PFAR is a promising cellular target for the treatment of prion diseases.

There is growing evidence that other proteinopathies share key biophysical and biochemical characteristics with prionopathies [16]. In this type of disease, affected proteins are for example $A \beta$ and Tau for Alzheimer's disease, and $\alpha$-synuclein for Parkinson's disease. Oculopharyngeal muscular dystrophy (OPMD) is also classified as a proteinopathy, in which poly(A) binding protein nuclear 1 protein (PABPN1) forms aggregates within the nuclei of patients' muscle cells $[17,18]$. OPMD is a late-onset, autosomal dominant genetic neuromuscular disease caused by a short expansion of a GCG repeat encoding a polyalanine tract located at the $\mathrm{N}$-terminus of PABPN1 [19]. Ten alanine residues are present in the normal protein and are expanded from 11 to 18 alanine residues in the mutant forms of the protein [20-22]. Similarly to polyglutamine expansion diseases, the triplet-expanded mutant PABPN1 protein aggregates and this is the pathological hallmark of OPMD. More important, we have previously shown that the antiPFAR drug GA was active in OPMD cells as well as Drosophila and mouse models [23, 24], suggesting that PFAR plays a role in PABPN1 aggregation in OPMD. These data highlight the fact that PFAR inhibitors may be beneficial for various proteinopathies, including prion diseases and OPMD.
As the prion field desperately lacks therapeutic solutions for affected patients, and since GA (hypotensive drug) as well as IQ (immune system activator used in epicutaneous administration) are not compatible with a chronic treatment targeting the CNS, we sought to identify other anti-prion compounds which are also approved drugs. Here, we show that flunarizine, an approved drug used to treat migraine, is also a potent anti-prion drug in cell culture. Flunarizine also shows a significant effect on the survival of intracerebrally $\mathrm{PrP}^{\mathrm{Sc}}$ infected mice. Following this observation, we employed an in silico approach based on similarities with the flunarizine chemical structure to identify other FDA-approved drugs that may also possess anti-prion activity and allow their therapeutic repositioning according to SOSA (selective optimization of side activities of drug molecules $[25,26]$ ) approach. This strategy is based on the screening of drugs that are already in the clinic or in clinical trials to determine if they could have biological activities - in our case, the inhibition of prion propagation - other than those initially described during their therapeutic development. This approach is particularly popular for rare or neglected diseases, as their therapeutic targets are not present in the safety or selectivity profiles in pharmaceutical companies. As their toxicity, safety and bioavailability in humans have already been tested, the drugs identified in these SOSA-based libraries may reach patients faster and may also be directly administered as potential compassionate therapeutics to people presenting symptoms of prion diseases. Using this approach, we have been able to identify 17 drugs approved by the FDA and/or other drug agencies, which are, to our knowledge, newly described anti-PrP ${ }^{\mathrm{Sc}}$ drugs, and provide a new perspective for their therapeutic repositioning as anti-prion compounds in the clinic. Among a selection of the most potent anti-prion drugs identified, we found that, like flunarizine, azelastine, duloxetine, ebastine, loperamide and metixene can inhibit $\mathrm{PrP}^{\mathrm{Sc}}$ propagation in organotypic mouse cerebellum slice cultures. From a mechanistic point of view, flunarizine and its six structural analogs were also found to be potent PFAR inhibitors, confirming our previous findings that PFAR is a relevant therapeutic target to curb prion propagation. As PFAR has also been shown to play a role in OPMD [23] and since there is no pharmacological treatment currently available for this disease, flunarizine and some of its structural analogs were also challenged for their capacity to reduce the phenotypes of OPMD in both cellular and Drosophila models. These new anti-PFAR molecules were able to reduce not only PABPN1 aggregation in cell culture but also muscular defects in OPMD Drosophila.

Altogether, we report here the identification of six new anti-PFAR FDA-approved drugs, namely azelastine, duloxetine, ebastine, flunarizine, loperamide and metixene as potent drug candidates for prion diseases as well as for OPMD. In addition, these data confirm our previous observations that PFAR plays a major role in prion propagation and toxicity of PABPN1 aggregation [23]. 


\section{Methods}

\section{Selection of Anti-prion Drug Candidates}

ROCS Shape-Based Virtual Screening The latest release of DrugBank (version 5.1.0, released 2018-04-02) was downloaded directly from https://www.drugbank.ca and filtered to only conserve FDA-approved or withdrawn drugs. Multiconformer files were generated by OMEGA v2.5.1.4 from OpenEye Scientific Software (http:/www.eyesopen. com/), leading to 1887 approved or withdrawn drugs saved in oeb.gz format. These generated multiconformational files were used as the input database for performing similarity search with Rapid Overlay of Chemical Structures (vROCS 3.2.2.2 from OpenEye Scientific Software (http://www. eyesopen.com/)). ROCS is designed to carry out large-scale 3D database searches. It performs similarity searches by using a shape- and/or pharmacophore-based superposition method that finds the similar but non-intuitive compounds. It uses only the heavy atoms of a ligand ignoring the hydrogens. The output files of the similarity search were then ranked according to their ComboScore, which is based on a combination of shape and pharmacophore similarity.

The structure of flunarizine used as query was extracted from experimentally crystallized structure [27] obtained from the Cambridge Crystallographic Data Centre via www.ccdc. cam.ac.uk/structures (CCDC identifier: JOBSIE).

Azelastine, biperiden, cinnarizine, diphenhydramine, epinastine, ethosuximide, flunarizine, imipramine, loperamide, orphenadrine, paramethadione, thioridazine, triflupromazine, zonisamide and $\mathrm{GuHCl}$ were purchased from Sigma-Aldrich. Astemizole, clemastine, duloxetine, ebastine and metixene were purchased from CarboSynth. Benzydamine and nefopam were purchased from LGC. Antazoline, atomoxetine, cetirizine, citalopram, diazepam, ketotifen and zimelidine were purchased from Tocris. Alimemazine, chloropyramine, dicyclomine, diphenidol, mirtazapine and prenylamine were purchased from Prestwick.

\section{PrPSc Clearance Assay in Cultured MovS6 Cells}

These experiments were performed as previously described [4, 5, 28]. Briefly, MovS6 cells chronically infected with ovine $127 \mathrm{~S}$ prion strain were plated in 6-well plates (Starlab, France), treated for 6 days with the indicated concentrations of candidate drugs and then lysed in a buffer containing $0.5 \%$ sodium deoxycholate, $0.5 \%$ Triton-X100 and $5 \mathrm{mM}$ Tris- $\mathrm{HCl}$ pH 7.5. Cell lysates $(250 \mu \mathrm{g})$ were digested by $20 \mu \mathrm{g} / \mathrm{mL}$ of proteinase K (PK, Thermo Scientific, Waltham, MA) to identify $\mathrm{PrP}^{\mathrm{Sc}}$ proteins which are resistant to proteolytic degradation. PK-treated homogenates were loaded on $10 \%$ Bis-Tris SDS-polyacrylamide gels (NuPAGE, Invitrogen/Thermo Scientific), and subjected to western blotting onto nitrocellulose membranes (GE Healthcare, Barrington, Illinois). For normalization purposes, $25 \mu \mathrm{g}$ of non-PKtreated homogenates was also loaded on $10 \%$ Bis-Tris SDSpolyacrylamide gels, and subjected to western blotting onto nitrocellulose membranes. PrP proteins were immunolabelled on PK-treated and non-PK-treated samples using an anti-PrP antibody (Sha31, 1/40,000, Bertin pharma, [29]). Loading accuracy was controlled by tubulin detection on non-PK-treated samples (YOL1/34, 1/3000, Abcam, Cambridge, UK).

\section{Mouse Lines and Ethical Statement}

We used mice overexpressing ovine PrP (tg338 line previously described [30]). All animal experiments were carried out in strict accordance with EU directive 2010/63 and were approved by the author's institution local ethics committee (Comethea, INRA Agroparitech ethics committee, permit number 12/034) and by the French Ministry of Education, higher education and research (authorization no. 4292). All efforts were made to minimize suffering.

\section{Drug Treatment of a Mouse Model for Prion Disease}

Experiments were performed as previously described [5, 31]. Briefly, thirty 8-week-old female mice overexpressing ovine $\operatorname{PrP}(\operatorname{tg} 338$ line [30]) were infected intracerebrally with $20 \mu \mathrm{L}$ of the $127 \mathrm{~S}$ scrapie strain at the $0.01 \%(w / v)$ dose, corresponding to $2 \times 10^{3.2} \mathrm{LD}_{50}$ (the infectious titre of $127 \mathrm{~S}$ in $\operatorname{tg} 338$ mouse brain is $10^{9.2} \mathrm{LD}_{50} / \mathrm{g}$ of brain, [32]). Forty days postinfection, 9 mice were intraperitoneally injected 3 times per week with a dose of $100 \mu \mathrm{L}$ at $4 \mathrm{mg} / \mathrm{mL}(20 \mathrm{mg} / \mathrm{kg})$ of flunarizine solubilized in $8 \%$ DMSO. As negative controls, 11 mice were intraperitoneally injected 3 times per week with $100 \mu \mathrm{L}$ of $8 \%$ DMSO. To avoid unethical suffering of prioninfected mice, the treatment was stopped around day 70 postinfection, when the first symptoms appeared in the control group of mice. Mice were euthanized at the terminal stage according to ethics rules. Spleens and brains were collected from 4 mice euthanized at the terminal stage of the disease and analysed for their $\mathrm{PrP}^{\mathrm{Sc}}$ content, as previously described [5, 6, 32].

\section{Luciferase Refolding Assay}

[psi-] ltv1 $\Delta /$ hsp104 $\Delta$ 74-D694 S. cerevisiae yeast strain $(\Delta \mathrm{L} \Delta \mathrm{H},[15])$ was transformed with pDCM90 plasmid [33, 34], allowing constitutive expression of temperature-sensitive luciferase polypeptide (LuxAB). Transformants were exponentially grown at $29^{\circ} \mathrm{C}$. Cells were treated with the indicated concentrations of drugs or DMSO $2 \mathrm{~h}$ before the heat shock. The $100 \%$ reference corresponds to luciferase activity measured just before the heat shock. Luciferase was then heatinactivated by incubation at $43.5{ }^{\circ} \mathrm{C}$ for $60 \mathrm{~min}$. 
Cycloheximide (10 $\mu \mathrm{g} / \mathrm{mL}$; Sigma-Aldrich, St. Louis, MO) was added after $45 \mathrm{~min}$ at $43.5^{\circ} \mathrm{C}$ to prevent luciferase synthesis during recovery. Luciferase activity was immediately measured after 60 -min post-heat shock $(0 \mathrm{~min})$ and cells were then left to recover at $29{ }^{\circ} \mathrm{C}$ for indicated times, luciferase activity being assessed at 90 and $150 \mathrm{~min}$ by adding $10 \mu \mathrm{L}$ n-decylaldehyde (Decanal, Sigma-Aldrich) to $120 \mu \mathrm{L}$ yeast culture. Luminescence was quantified using a Varioscan microplate reader (Thermofisher). Luciferase activity at 0-, 90-, and 150-min recovery was then expressed as a percentage of the activity before the heat treatment for each strain (100\%).

\section{Drug Treatment of Prion-Infected Cultured Organotypic Cerebellar Slices}

The anti-prion activity of drugs was evaluated using cultured organotypic cerebellar slices (OCSs), as described in [4] using $\operatorname{tg} 338$ transgenic mice overexpressing the VRQ allele of the ovine prion protein [31]. The preparation and culture of slices were performed as described in [4, 35] using insert (Millipore), except for prion infection which was performed the day of cerebellum slicing for $1 \mathrm{~h}$ on ice in 24-well plates (Starlab) with $50 \mu \mathrm{g} / \mathrm{mL}$ of a brain stock prepared from terminally ill tg 338 mice experimentally infected with $127 \mathrm{~S}$ prion strain. Seven days after infection, the drugs were added to the cerebellum slices at indicated concentrations. As a negative control, vehicle only (DMSO) was added. The slices were then cultured for an additional 21 days before harvesting. Fresh drugs were added at each medium change, 3 times per week, at indicated concentrations.

Pools of slices (at least seven slices/condition) were harvested by scrapping and homogenized in $350 \mu \mathrm{L}$ of lysis buffer $(0.5 \%$ sodium deoxycholate, $0.5 \%$ Triton-X100, $5 \mathrm{mM}$ Tris- $\mathrm{HCl} \mathrm{pH}$ 7.5) with the Beadbug homogenizer (Benchmark Scientific, Sayreville, NJ) as previously described [4]. Slice lysates $(100 \mu \mathrm{g})$ were treated by $20 \mu \mathrm{g} / \mathrm{mL}$ proteinase $\mathrm{K}$ (PK, Thermo Scientific) for $30 \mathrm{~min}$ at $37^{\circ} \mathrm{C}$. For the analysis, PK-treated slice homogenates and $25 \mu \mathrm{g}$ nonPK-treated slice homogenates were denatured for $10 \mathrm{~min}$ at $95{ }^{\circ} \mathrm{C}$ and then loaded on $10 \%$ SDS-polyacrylamide gels (NuPAGE, Invitrogen) and electrotransferred onto nitrocellulose membranes (GE Healthcare). PK-treated and non-PKtreated samples were immunoblotted with anti-PrP antibody (Sha31, 1/40,000, Bertin Pharma, Montigny-le-Bretonneux, France). Loading accuracy was controlled by immunoblotting $25 \mu \mathrm{g}$ of non-PK-treated homogenates with anti-tubulin antibody (YOL1/34, 1/3000, Abcam).

\section{Assessment of the Action of Drugs on Nuclear Aggregate Number in an OPMD Cell Line}

The OPMD mutated PABPN1 (Ala17) stable cell line [36] was cultivated on a matrigel-coated surface ( $\mu$-slide 8 wells ibidis, Biovalley) in DMEM medium supplemented with $20 \%$ of fetal calf serum (Invitrogen), $0.5 \%$ of chick embryo extract (MP Biomedicals, Irvine, CA), $100 \mathrm{U} / \mathrm{mL}$ penicillinstreptomycin antibiotic (Thermofisher), $500 \mu \mathrm{g} / \mathrm{mL}$ geneticin (G418, Life Technologies, Carlsbad, CA) and $10 \mathrm{U} / \mathrm{mL}$ interferon gamma (Millipore/Merck, Darmstadt, Germany) at $33{ }^{\circ} \mathrm{C}$ in a humidified $5 \% \mathrm{CO}_{2}$ air atmosphere. At $80 \%$ confluence, Ala17 cells were differentiated in DMEM supplemented with $10 \%$ horse serum and $1 \%$ penicillinstreptomycin at $37{ }^{\circ} \mathrm{C}$ in a $5 \% \mathrm{CO}_{2}$, humidified atmosphere.

At 2 days of differentiation, half of the differentiation medium was replaced with fresh differentiation medium containing the drugs solubilized in DMSO at indicated concentrations. Two days later, cells were washed once in phosphate-buffered saline (PBS) and fixed for 15 min with $4 \%$ paraformaldehyde for PABPN1 immunostaining. Cells were then incubated for $15 \mathrm{~min}$ in PBS-0.1 M glycine and permeabilized in blocking buffer containing 3\% BSA, and 5\% Goat serum in PBS- $0.2 \%$ Triton X-100. Anti-PABPN1 primary antibody $(1 / 200$, ab75855, Abcam) was incubated $1 \mathrm{~h}$ at room temperature in blocking buffer. Cells were washed five times with PBS-0.1\% Triton X-100 prior incubation with goat anti-rabbit secondary antibody conjugated to Alexa Fluo 488 (Life Technologies) together with Phalloidin 555 (Interchim, Montluçon, France). Cells were then washed five times with PBS-0.1\% Triton X-100, and stained with Hoechst for 10 min at room temperature. Pictures were taken with the $\times 40$ objective and five to seven hundred nuclei were counted for each condition.

\section{OPMD Drosophila Treatment}

The Drosophila OPMD model contains an alanine-expanded mammalian PABPN1 cDNA (PABPN1-17ala) that is specifically and constitutively expressed in adult indirect flight muscles from the Act88F-PABPN1-17ala transgene [37]. Drug-supplemented food was prepared as follows: instant Drosophila medium (Carolina Biological Supply Company, Whitsett, NC) was reconstituted in each vial with a solution of $1 \%$ yeast in water, supplemented with indicated concentrations of either drug solubilized in DMSO or DMSO alone. Each vial contained $2 \mathrm{~mL}$ of reconstituted medium for twenty flies per vial. The flies were put on freshly prepared medium every day. Abnormal wing posture was quantified every day by pooling males by batches of five in an empty vial and scoring abnormal wing position by direct observation of the flies through the vials, without anaesthesia.

\section{Results}

\section{Flunarizine Is a Potent Anti-prion Drug}

Flunarizine (Fig.1a) was identified in our laboratory through a yeast-based screening strategy and was shown to reduce the 
a

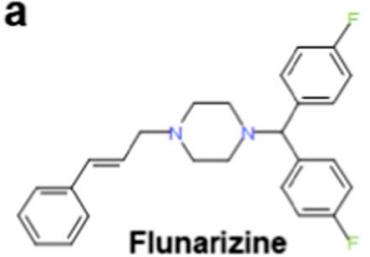

b

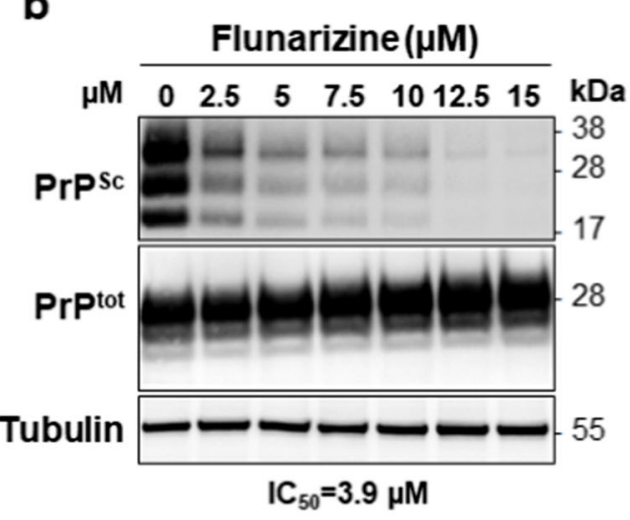

C

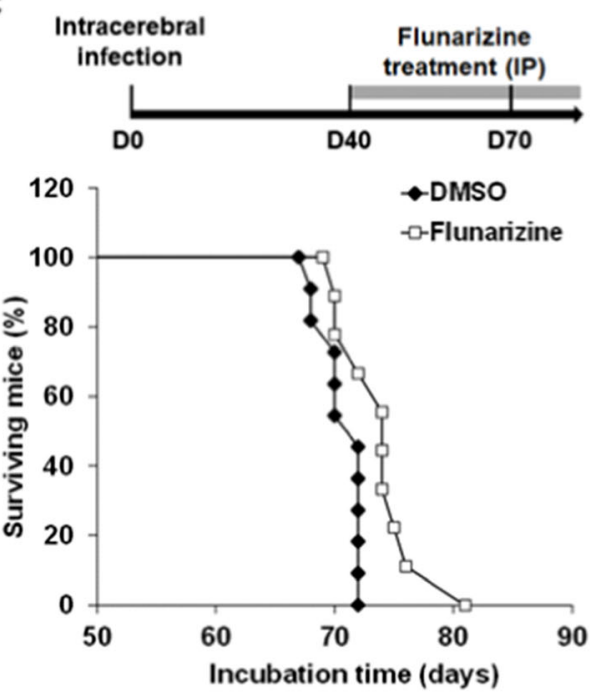

Fig. 1 Flunarizine is a potent anti-prion drug. Flunarizine was identified through a yeast-based screening strategy [7]. (a) Structure of flunarizine. (b) Flunarizine activity against $\operatorname{PrP}^{\mathrm{Sc}}$ in chronically prion-infected MovS6 cells. Scrapie-infected MovS6 cells (127S prion strain, [28]) were treated for 6 days with a range of flunarizine concentrations. Cell lysates were then subjected to $\mathrm{PK}$ digestion to specifically reveal $\mathrm{PrP}^{\mathrm{Sc}}$ by immunoblot. The effect of flunarizine on the steady state level of $\operatorname{PrP}\left(\mathrm{PrP}^{\text {tot }}\right)$ was determined on the same MovS6-treated cell lysates in the absence of PK treatment (lower panel). The ratios of western blot $\mathrm{PrP}^{\mathrm{res}} / \mathrm{PrP}^{\mathrm{tot}}$ signals are presented in the form of histograms below the blot. The same blot was used to check the loading homogeneity using an anti-tubulin antibody (bottom panel). The blot shown is representative of at least two independent experiments which all produced similar results. Flunarizine $\mathrm{IC}_{50}$ is $3.9 \mu \mathrm{M}$. (c) Survival of intracerebrally infected mice treated with flunarizine. Tg338 transgenic mice overexpressing ovine $\operatorname{PrP}$ were intracerebrally infected with scrapie (strain $127 \mathrm{~S}$ ). Forty days post-infection, eleven mice were treated with DMSO as control, and nine mice were treated three times a week with $20 \mathrm{mg} / \mathrm{kg}$ of flunarizine solubilized in DMSO, until the appearance of the first clinical signs in mice from the control group at day 70 , as illustrated by the top scheme. $p<0.0146$, Kruskal-Wallis test amount of $\mathrm{PrP}^{\mathrm{Sc}}$ in chronically prion-infected MovS6 cells, with an $\mathrm{IC}_{50}$ of $3.9 \mu \mathrm{M}$ (Fig. 1b). Flunarizine is a selective calcium entry blocker as well as an antagonist of histamine $\mathrm{H} 1$ receptor that readily crosses the blood-brain barrier [38]. This drug is used in many developed countries (except USA, UK and Japan) to treat migraine headaches, occlusive peripheral vascular disease and vertigo of central and peripheral origin, and as an adjuvant in the therapy of epilepsy [38-42].

We next challenged the effect of flunarizine using an in vivo model of prion infection. We monitored the survival of $\operatorname{tg} 338$ mice intracerebrally infected with a relatively high dose of $127 \mathrm{~S}$ prion at day 0 , and treated by $20 \mathrm{mg} / \mathrm{kg}$ flunarizine three times per week from 40 days post-infection onward. Mice treated by flunarizine survived significantly longer than DMSO-treated mice $(p<0.0146$, Kruskal-Wallis test) with a mean survival time of the flunarizine group mice increased by approximately $6 \%$ compared to the control group ( $n=9$ for flunarizine and $n=11$ for DMSO, Fig. 1c). These results confirm that flunarizine is a potential anti-prion drug.

\section{Identification of New Approved Anti-prion Drugs Based on Flunarizine Structure}

In order to broaden the range of new anti-prion drugs that could potentially be used to treat patients with prion diseases, and considering that flunarizine might not be the most optimized anti-prion drug, we performed an in silico screening, ranking the 1887 FDA-approved or withdrawn drugs present in the Drugbank database (https://www.drugbank.ca/) for their structural similarities with flunarizine. In terms of pharmacophoric pattern, flunarizine is composed of three aromatic moieties, as well as two protonable nitrogen atoms, which can interact with specific biological targets through electrostatic or H-bond acceptor interactions (Fig. 2a). We chose to use the ROCS method from OpenEye Scientific, using a shape- and/or pharmacophore-based superposition method that finds similar but non-intuitive compounds. FDA-approved drugs exhibiting similarities with flunarizine were then ranked according to the scoring function ComboScore, which is based on a combination of shape and pharmacophore similarities. The resulting ranking was a manner of classifying the FDA-approved drugs according to their similarities with flunarizine (Table S1). As flunarizine is known to be a non-selective calcium channel blocker with antihistamine activity as well as other actions including serotonin receptor and dopamine D2 blocking activities, it was not surprising to find in the top 100 several compounds targeting histamine, muscarinic and dopamine, as well as both serotonin receptors and transporters.

From the ranking shown in Table S1, a series of thirty molecules was selected throughout the classification. According their ROCS ranks, we chose compounds exhibiting a high or a medium level of similarity with flunarizine, short- 
a

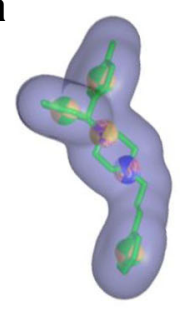

b
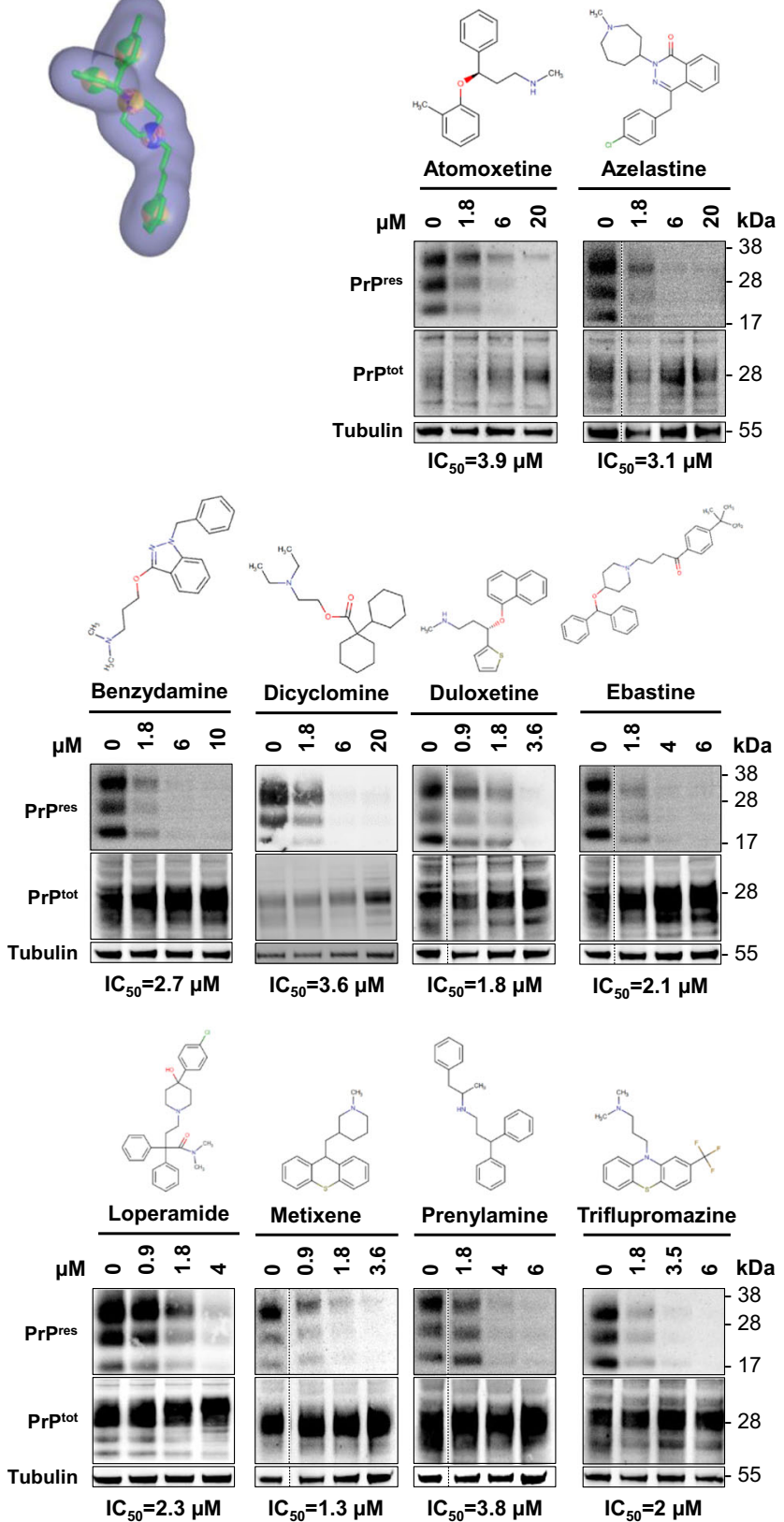

Fig. 2 Anti-prion activity of seventeen structural analogs of flunarizine. (a) Five-site pharmacophore model of flunarizine containing two nitrogen atoms (pink/yellow and blue/pink), and three aromatic rings (green/yellow). (b) Anti-prion activity of the ten most active $\left(\mathrm{IC}_{50} \leq 4 \mu \mathrm{M}\right)$ flunarizine structural analogs using scrapie-infected MovS6 cells as described in Fig. 1. Cells were treated for 6 days with indicated drug concentrations, lysed and then subjected to PK digestion to specifically reveal PK-resistant $\operatorname{PrP}^{\mathrm{Sc}}\left(\mathrm{PrP}^{\mathrm{res}}\right)$ by immunoblot. Structures of molecules and $\mathrm{PrP}^{\mathrm{Sc}}$ immunoblots of MovS6-treated cells are presented on the top of each western blot. Drug-treated samples and their corresponding DMSO control were processed on the same immunoblot. The blots shown are representative of at least two independent experiments which produced similar results. The effect of compounds on the steady-state level of PrP $\left(\mathrm{PrP}^{\mathrm{tot}}\right)$ was determined on the same non-PK-treated cell lysates (middle panel). The same blot was used to check the loading homogeneity using an anti-tubulin antibody (bottom panel). The $\mathrm{IC}_{50}$ of each drug is indicated below the blot listing compounds sharing targets with flunarizine, such as H1 receptor, as well as compounds targeting cellular pathways common or not with flunarizine (Table 1 and Table S2). In other words, we consider the ROCS ranking as a guideline to classify approved drugs exhibiting more or less structural similarities with flunarizine, and combine that with a diversity of addressed biological targets in order to select these thirty compounds. To evaluate the potential involvement of calcium channels in $\mathrm{PrP}^{\mathrm{Sc}}$ propagation, three calcium antagonists were selected, although they are not closely related to the calcium antagonist flunarizine (ranking > 500) (ethosuximide, paramethadione, zonisamide, Table S2). Altogether, the thirty selected FDA-approved drugs represent thirteen different cellular targets (Table S2). In addition to the thirty selected drugs resulting from our similarity screening approach, we also selected three reference compounds, namely astemizole [43-45], clemastine [44] and thioridazine [44, 46] which have previously been shown to inhibit prion propagation (labelled \# in Table 1; Table S1).

We first evaluated the anti-PrP $\mathrm{P}^{\mathrm{Sc}}$ activity of the thirty selected structural analogs of flunarizine using the MovS6 cell line infected by the $127 \mathrm{~S}$ scrapie prion strain [30]. We showed that seventeen out of the thirty tested drugs showed anti-PrP $\mathrm{P}^{\mathrm{Sc}}$ activity, ten of which with an $\mathrm{IC}_{50} \leq 4 \mu \mathrm{M}$ (Fig. 2b) and 7 with an $\mathrm{IC}_{50}$ between 4 and $20 \mu \mathrm{M}$ (Fig. S2), without affecting $\mathrm{PrP}^{\text {tot }}$ level (Fig. $2 \mathrm{~b}$ and Fig. S2). Thirteen out of the thirty selected drugs were inactive ( $\mathrm{IC}_{50} \geq 20 \mu \mathrm{M}$; Fig. S3). Overall, this approach allowed us to identify seventeen new potential anti-prion FDA-approved drugs.

\section{Flunarizine and Some of Its Structural Analogs Are Active Against PrP ${ }^{\mathrm{Sc}}$ in Organotypic Cerebellar Slice Culture}

We next evaluated the anti-prion activity of the 7 most active newly identified drugs (azelastine, duloxetine, ebastine, flunarizine, loperamide, metixene and triflupromazine) on organotypic cerebellum slice culture (OSC), a relevant alternative to animal experimentation which has been shown to closely mimic prion replication and propagation on a structured organ in which cohesion between cells and tissues is maintained [35]. The selected FDA-approved drugs are marketed to target different biological targets (GCPRs: H1, $\mu$-opioid, 5-HT, D2, M1; transporters: SERT and NET; channels: VSCC). Cerebellum slices derived from ovine PrP transgenic mice (tg338 line) and infected with $127 \mathrm{~S}$ prions [47] were treated for 21 days by the indicated concentrations of the drugs, 7 days post-infection. Tested at doses ranging from 20 to $35 \mu \mathrm{M}$, azelastine, duloxetine, ebastine, flunarizine, loperamide, metixene and the reference drug astemizole showed anti-PrP ${ }^{\mathrm{Sc}}$ activity in OSC (Fig. 3), whereas triflupromazine and the reference drugs clemastine and thioridazine showed no activity. None of the active drugs except 
Table 1 Anti-PrP ${ }^{\mathrm{Sc}} \mathrm{IC}_{50}$ and ROCS ranks of flunarizine, the 30 selected flunarizine structural analogs and the three anti-prion references. For each drug, $\mathrm{IC}_{50}$ against $\mathrm{PrP}^{\mathrm{Sc}}$ in cell culture are indicated, together with ROCS ranks. \# indicates drugs previously described as anti-PrP $\mathrm{P}^{\mathrm{Sc}}, *$ indicates withdrawn drugs

\begin{tabular}{llllllll}
\hline & Drugs & IC $_{50}$ & ROCS ranks & & Drugs & IC $_{50}$ & ROCS ranks \\
\hline 1 & Alimemazine & 11.4 & 197 & 18 & Ethosuximide & Neg & 1471 \\
2 & Antazoline & Neg & 129 & 19 & Flunarizine & 3.9 & 1 \\
3 & Atomoxetine & 3.9 & 41 & 20 & Isradipine & Neg & 327 \\
4 & Azelastine & 3.1 & 140 & 21 & Ketotifen & Neg & 78 \\
5 & Benzydamine & 2.7 & 368 & 22 & Loperamide & 2.3 & 25 \\
6 & Biperiden & 9.2 & 33 & 23 & Metixene & 1.3 & 101 \\
7 & Cetirizine & Neg & 10 & 24 & Mirtazapine & Neg & 225 \\
8 & Chloropyramine & 18.5 & 133 & 25 & Nefopam & Neg & 471 \\
9 & Cinnarizine & Neg & 2 & 26 & Orphenadrine & 9.1 & 16 \\
10 & Citalopram & 10.4 & 23 & 27 & Paramethadione & Neg & 1475 \\
11 & Diazepam & Neg & 595 & 28 & Prenylamine* & 3.8 & 6 \\
12 & Dicyclomine & 3.6 & 112 & 29 & Triflupromazine & 2 & 256 \\
13 & Diphenhydramine & 14.8 & 21 & 30 & Zimelidine* & 14.3 & 149 \\
14 & Diphenidol & $\mathrm{Neg}$ & 24 & 31 & Zonisamide & Neg & 1216 \\
15 & Duloxetine & 1.8 & 69 & 32 & Astemizole*\# & 0.5 & 36 \\
16 & Ebastine & 2.1 & 14 & 33 & Clemastine & 2 & 27 \\
17 & Epinastine & Neg & 683 & 34 & Thioridazine & 1.7 & 159 \\
\end{tabular}

loperamide affected the level of $\operatorname{Pr}^{\text {tot }}$. Altogether, these data indicate the potency of azelastine, duloxetine, ebastine, flunarizine, loperamide and metixene to reduce $\operatorname{PrP}^{\mathrm{Sc}}$ burden and highlight their therapeutic potential.

\section{Flunarizine and Its Structural Analogs Are PFAR Inhibitors}

We next wondered if the known targets of our selected active FDA-approved drugs could be involved in their anti-PrP ${ }^{\mathrm{Sc}}$ activity. Despite the fact that some of them are not used for their H1 receptor antagonist property, seven out of the seventeen active drugs identified were able to antagonize histamine H1 receptor, suggesting that histamine H1 receptor could be involved in $\mathrm{PrP}^{\mathrm{Sc}}$ clearance. We challenged this hypothesis by comparing the binding to $\mathrm{H} 1$ ( $\mathrm{H} 1$ binding $\mathrm{IC}_{50}$ from the literature) with the anti-PrP ${ }^{\mathrm{Sc}} \mathrm{IC}_{50}$ of flunarizine and its 30 selected structural analogs. The three reference drugs astemizole, clemastine and thioridazine were also included. We found no correlation between $\mathrm{H} 1$ affinity and anti-PrP ${ }^{\mathrm{Sc}}$ activity (Fig. S4). The fact that flunarizine was identified in an assay based on $S$. cerevisiae yeast cells that lack known homologues of histamine receptors [48] is an additional argument indicating that the $\mathrm{H} 1$ receptor is not involved in the anti-prion effect of the drug tested. Finally, since 5 out of the 13 inactive compounds tested also bind to $\mathrm{H} 1$ receptor, we concluded that $\mathrm{H} 1$ receptor is unlikely to be involved in $\operatorname{PrP}^{\mathrm{Sc}}$ clearance. Altogether, this set of thirty-three anti-prion compounds binds to sixteen different cellular targets (Fig. S5), suggesting that no common mechanisms can explain their capacity to clear $\mathrm{PrP}^{\mathrm{Sc}}$.
As we recently identified PFAR as being involved in $[\mathrm{PSI}+]$ prion propagation in yeast [15], we sought to determine whether flunarizine and its structural analogs could also inhibit PFAR. To this aim, we monitored PFAR inhibition using a luciferase refolding assay in yeast, which measures renaturation of a heat-denatured luciferase over time [33]. We performed this assay in PFAR-enriched yeast cells that were deleted for the protein chaperon Hsp104, and that have previously been shown to be particularly suitable to monitor PFAR inhibition [15]. The PFAR assay is based on $S$. cerevisiae cells which are much less permeable to drugs than mammalian cells. However, as yeast cell permeability is different for each drug, the tested concentrations had to be individually adapted to each drug $[4,5,14]$. Yeast cells expressing luciferase were heat-shocked to unfold luciferase, and cycloheximide was added to inhibit translation and avoid de novo luciferase synthesis, in order to only monitor luciferase refolding by PFAR over time (Fig. S6). For each drug, a range of concentrations was tested. Luciferase activity remained basal in the presence of $45 \mu \mathrm{M}$ ebastine, $100 \mu \mathrm{M}$ metixene, $150 \mu \mathrm{M}$ flunarizine, loperamide, duloxetine and azelastine, as well as $50 \mu \mathrm{M}$ of the reference compound astemizole, indicating that these molecules are potent PFAR inhibitors (Fig. 4). On the contrary, antazoline and diazepam, which showed no antiprion activity in cultured MovS6-infected cells (Fig. S3), were unable to inhibit PFAR (Fig. 4). Altogether, these data indicate that flunarizine and six of its anti-prion structural analogs astemizole, azelastine, duloxetine, ebastine, loperamide and metixene are PFAR inhibitors and share a common mechanism explaining their anti-prion activity. 


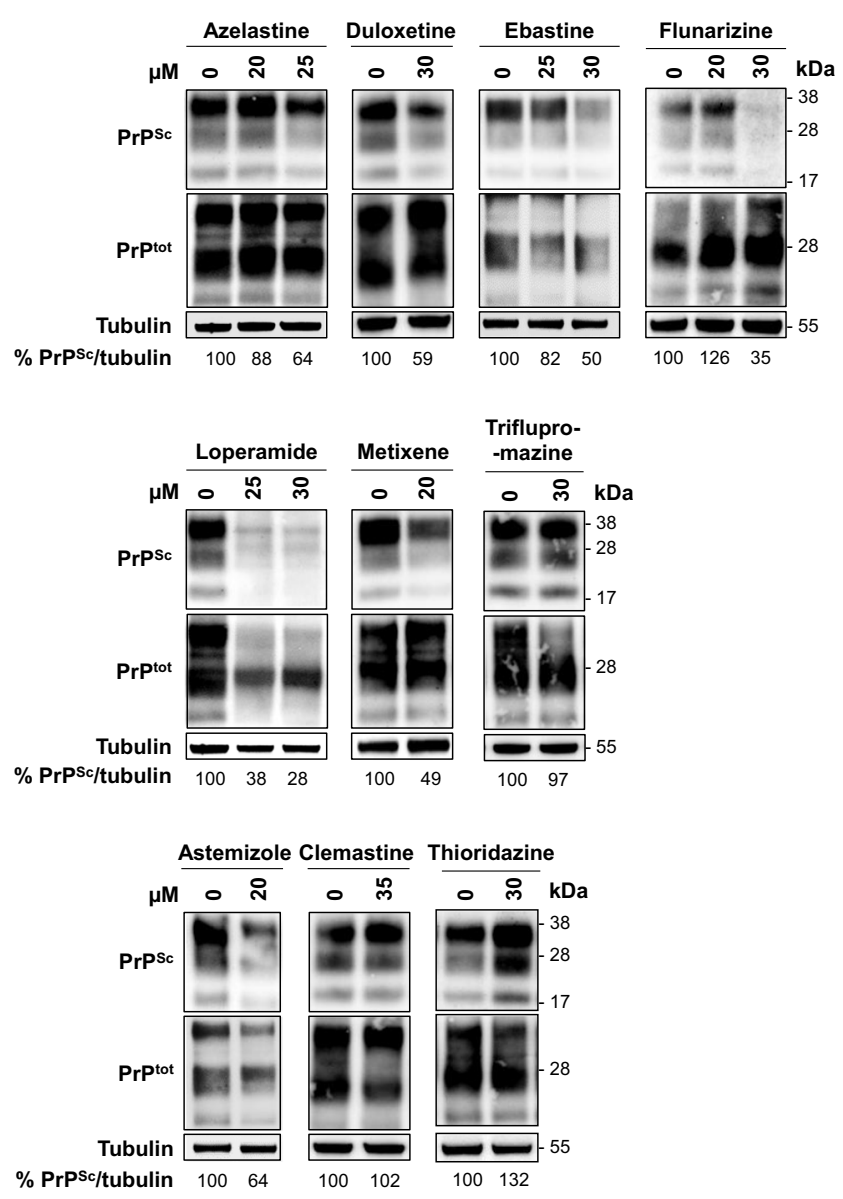

Fig. 3 Activity of flunarizine and 9 of its structural analogs on prioninfected organotypic cerebellar slice culture. Cerebellar slices from P9 to P12 tg338 transgenic mice were infected with $127 \mathrm{~S}$ ovine prion strain. Seven days after infection, slices were treated with indicated concentrations of drugs for 21 days. Infected cerebellar slice lysates were subjected to $\mathrm{PK}$ digestion to specifically reveal $\mathrm{PrP}^{\mathrm{Sc}}$ by western blot. The effect of compounds on the steady-state level of $\operatorname{PrP}\left(\mathrm{PrP}^{\text {tot }}\right)$ was determined on the same slice lysates in the absence of PK treatment (middle panel). The same blot was used to check the loading homogeneity using an antitubulin antibody (bottom panel). The blots shown are representative of two to four independent experiments which all produced similar results. Ratios of western blot $\mathrm{PrP}^{\mathrm{Sc}} /$ tubulin signals are indicated below each lane

\section{Flunarizine Is Active in OPMD Cellular and Drosophila In Vivo Models}

We previously showed that anti-PFAR and anti-prion drugs 6AP and GA efficiently decreased muscle degeneration in an OPMD Drosophila model, and had a synergistic effect with partial deletions of the DNA locus encoding rRNA that bear PFAR [23]. In addition, GA was also shown to reduce the size and number of PABPN1 nuclear aggregates in an OPMD Ala17 muscle cell model and improve muscle force in vivo in an OPMD mouse model [24]. Together, these data suggest that PFAR might also play a role in PABPN1 aggregation in OPMD. We therefore tested the efficiency of 10 of the newly identified anti-PFAR drugs in the OPMD Ala17 muscle cell model. When differentiated, this myogenic cellular model expresses PABPN1-Ala17, resulting in the formation of a large number of nuclear aggregates [36, 49]. Drugs were added to the cells at day 2 of differentiation and maintained in the medium for $48 \mathrm{~h}$. As $10 \mu \mathrm{M}$ of GA was previously shown to significantly reduce the number and size of PABPN1-Ala17 aggregates in Ala17 differentiated cells [24], GA was used as a positive control. Similarly, anti-PFAR drugs were tested at $10 \mu \mathrm{M}$. In these conditions, treatment with $10 \mu \mathrm{M}$ of flunarizine, metixene, thioridazine, astemizole, loperamide, duloxetine, azelastine and ebastine led to a significant reduction in the number of PABPN1-Ala17 aggregates (Fig. 5a), without altering cell differentiation. Moreover, flunarizine, metixene, thioridazine, loperamide and ebastine showed a better efficiency than GA (Fig. 5a) [24]. However, clemastine and triflupromazine, which showed no anti- $\mathrm{PrP}^{\mathrm{Sc}}$ activity in OSC, also showed no significant reduction in the number of PABPN1-Ala17 aggregates, similarly to the negative control diazepam which showed neither anti-PFAR nor anti-prion activity (Fig. 5a).

As flunarizine, metixene and ebastine induced a strong reduction in PABPN1 aggregates in OPMD cells and were more efficient than GA (Fig. 5a), we next tested their effect in the OPMD Drosophila model. This model recapitulates the disease characteristics, namely progressive muscle weakness and degeneration, and formation of PABPN1 nuclear aggregates $[37,50]$. Specifically, PABPN1-17ala expression in muscles leads to abnormal wing posture (Fig. S7a) resulting from affected indirect flight muscle function and muscle degeneration [23, 37]. The relevance of the Drosophila model has been validated by the fact that the molecular mechanisms identified as participating in OPMD in Drosophila have been confirmed in OPMD patient biopsies [37, 51]. As $2 \mathrm{mM} \mathrm{GA}$ was previously shown to significantly reduce the wing position defects of OPMD Drosophila when provided in the food from day 2 of adulthood [23], anti-PFAR drugs were also tested at $2 \mathrm{mM}$. However, at this concentration, flunarizine and ebastine showed toxicity. These two drugs were thus further used at $1.5 \mathrm{mM}$, concentration at which both drugs showed no toxicity (Fig. S7b). Drugs were administered to adult flies orally in the food at the indicated concentrations from day 2 to day 5 , and fresh food with drug was provided every day. Wing posture defects were recorded from day 3 to day 6 of adulthood. The effect of drugs was quantified by recording the number of PABPN1-17ala-expressing flies with abnormal wing posture. Flunarizine, metixene and ebastine showed a beneficial effect with a significant decrease in the number of flies with abnormal wing posture compared to DMSO alone (Fig. 5b), without affecting Drosophila survival (Fig. S7b). Altogether, these data demonstrate that these new anti-PFAR drugs are active in alleviating defects in OPMD cell and Drosophila models. This indicates that these drugs might have a therapeutic potential for OPMD patients. 

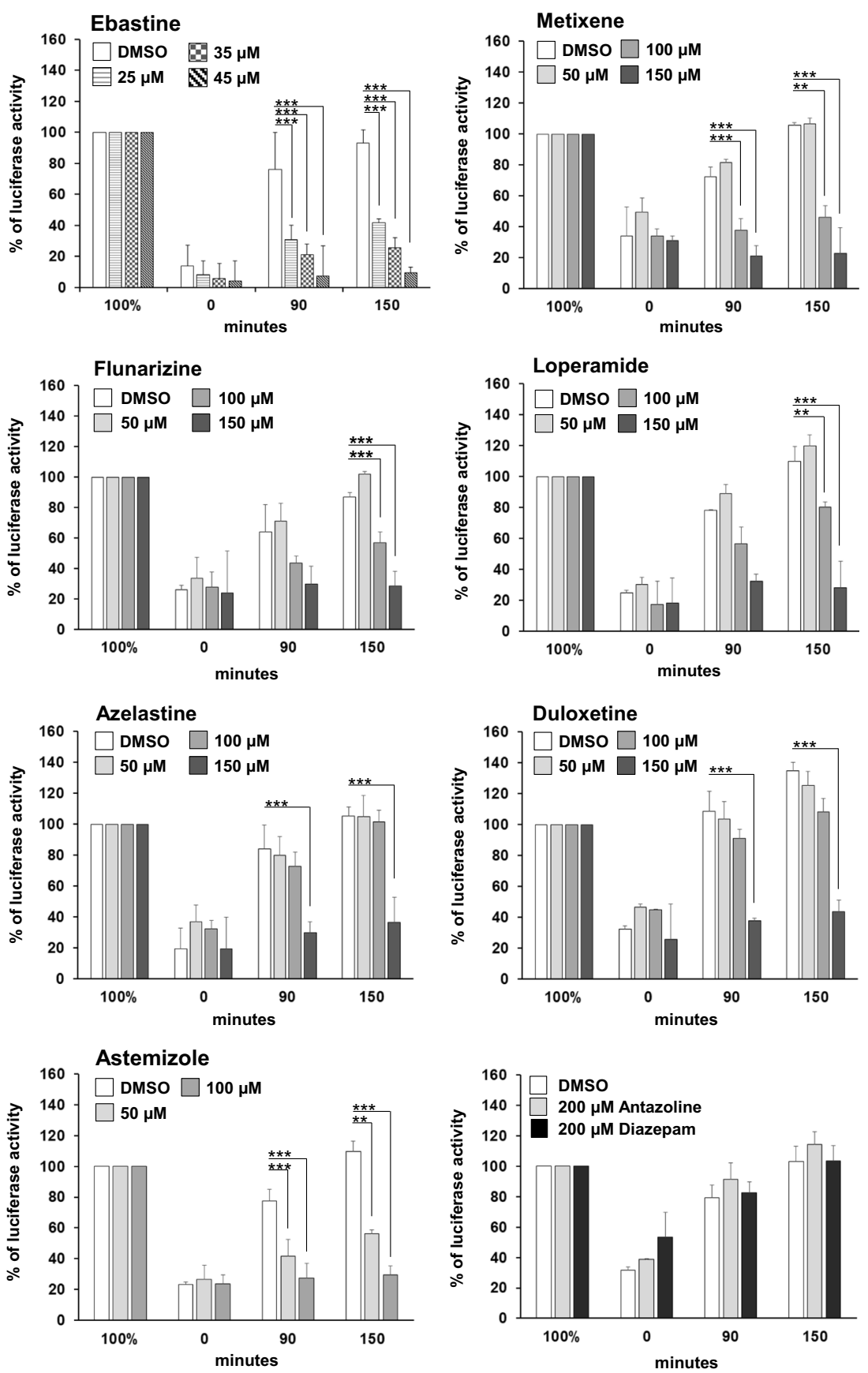

Fig. 4 PFAR activity monitoring in the presence of flunarizine and six of its most active structural analogs. The effect of astemizole, azelastine, duloxetine, ebastine, flunarizine, loperamide and metixene was tested on PFAR using a luciferase refolding assay in yeast cells (Fig. 4- Fig. S6). The heat shock reduced luciferase activity down to 20 to $30 \%$ of its initial level. In the presence of DMSO, luciferase activity increased over time (white bars), which indicates that it recovers its active conformation thanks to PFAR. In the presence of increasing concentrations of antiPFAR drugs, luciferase activity recovery is less and less efficient. Ebastine showed a strong anti-PFAR activity from $25 \mu \mathrm{M}$, astemizole

\section{Discussion}

We started this study by identifying flunarizine as an anti-prion drug in yeast and mammalian cells. We next established a proof

from $50 \mu \mathrm{M}$, metixene and flunarizine from $100 \mu \mathrm{M}$, and loperamide, azelastine, and duloxetine from $150 \mu \mathrm{M}$. In the presence of antazoline and diazepam, luciferase activity increased over time as in the presence of DMSO, which indicates these two drugs do not inhibit PFAR, which is in good correlation with the fact they are inactive against $\mathrm{PrP}^{\mathrm{Sc}}$. Experiments were repeated two to three times. A representative assay including three technical repeats is shown with error bars. Bar height represents the mean relative to DMSO-treated cells at each time point; ${ }^{* *} p$ value $<0.01$ or $* * * p<0.001$, ANOVA one way

of principle that flunarizine exerts an anti-prion activity ex vivo and in vivo. The mice survival was significantly improved, despite the use of a very stringent transgenic mouse model, which overexpresses 8-fold ovine PrP and was inoculated by 


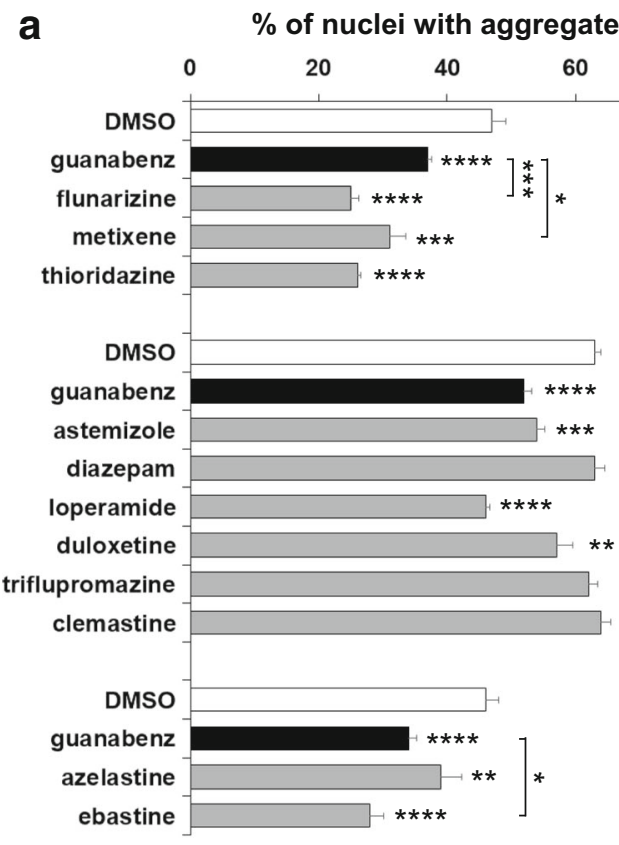

Fig. 5 Anti-PFAR drugs are active in OPMD cellular and Drosophila models. (a) Effect of flunarizine and nine of its structural analogs on PABPN1 nuclear aggregates in OPMD muscle cells during differentiation. OPMD cells were plated and left to differentiate. At 2 days of differentiation, $10 \mu \mathrm{M}$ drugs were added to the medium at indicated concentrations. At 4 days of differentiation, the myotubes were fixed, permeabilized and stained with PABPN1. Since the percentage of nuclei containing aggregates in the OPMD Ala17 cells is linked to the differentiation status of the cells $[36,49]$, the values obtained with DMSO differ between experiments. Therefore, for each independent experiment, DMSO and GA (10 $\mu \mathrm{M}$, positive control) were always added as internal controls to compare from one experiment to another the percentage of nuclei containing aggregates. For each condition, 500 to 700 nuclei were

the intracerebral route with a relatively high dose of prion. Furthermore, the treatment was initiated at the late stage of the disease and was administered by the intraperitoneal route. b

80
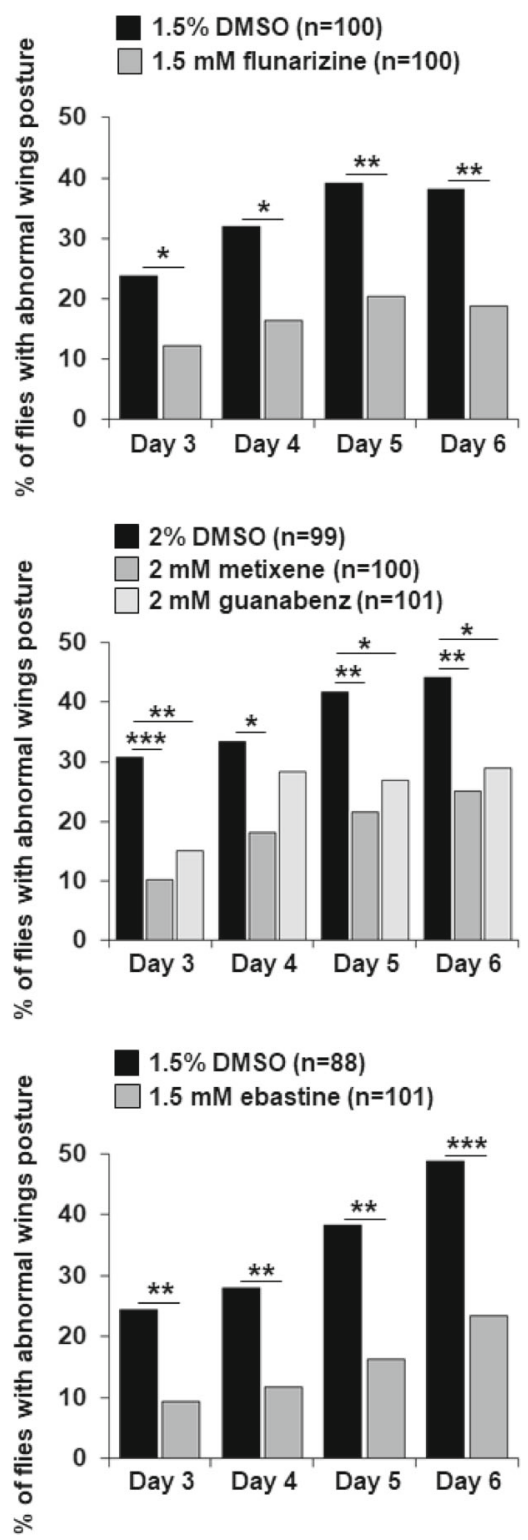

counted. Diazepam $(10 \mu \mathrm{M})$ was used as a negative control. Values are expressed as means \pm SD compared to DMSO $(* * p$ value $<0.01$, $* * * p<0.001, * * * * p<0.0001$, unpaired $t$ test) or GA (* $p$ value $<0.05$, $* * * p<0.001$, Turkey's multiple comparisons test). (b) Effect of flunarizine, metixene and ebastine on the wing posture defect in the Drosophila OPMD model (Fig. 5-Figure Supplement 1). Quantification of PABPN1-17ala-expressing flies (Act88F-PABPN1-17ala/+) showing wing position defects. Flies were fed either with DMSO or with the indicated concentration of flunarizine, metixene or ebastine at $25^{\circ} \mathrm{C}$. GA was used as a positive control. Feeding with GA or the three tested compounds led to a decreased number of flies with abnormal wing posture. $* p$ value $<0.05, * * p<0.01$ and $* * * p<0.001$ using the chi-square test

Based on this promising result, we decided to look for other FDA-approved drugs and used a strategy mixing structural similarities with flunarizine (ROCS ranking) and diversity in the 
addressed biological targets, leading to the selection of thirty drugs. The anti-prion activity of the selected molecules was mainly correlated with their ROCS ranking, as $76 \%$ of the selected drugs showing an anti-prion activity have a rank $\leq 200$ (Fig. S8). These observations indicate that our screening strategy was relevant to select molecules active against $\mathrm{PrP}^{\mathrm{Sc}}$ and it is possible that there are still some undescribed anti-prion drugs present in the top two hundred drugs shown in Table S1.

Thanks to this approach, we report here the identification of seventeen new anti-prion drugs that are FDA-approved for other pathologies and that could be readily usable to treat patients with prion diseases for whom no treatment is currently available. Notably, flunarizine and ten of these newly identified anti-prion drugs, namely atomoxetine, azelastine, benzydamine, dicyclomine, duloxetine, ebastine, loperamide, metixene, prenylamine and triflupromazine, have an $\mathrm{IC}_{50} \leq$ $4 \mu \mathrm{M}$ in mammalian cell culture. We further showed that azelastine, duloxetine, ebastine, flunarizine, loperamide and metixene are active against $\mathrm{PrP}^{\mathrm{Sc}}$ in organotypic mouse cerebellum slice culture, thus emphasizing the therapeutic potential of these drugs for the treatment of prion diseases.

While we have identified anti-prion drugs exhibiting structural similarities, we may also have found a common mechanism explaining their anti-prion activity. Indeed, flunarizine and six of its most potent anti-prion structural analogs (astemizole, azelastine, duloxetine, ebastine, loperamide and metixene) are potent inhibitors of PFAR, a protein folding activity born by rRNA which has previously been shown to be involved in $[P S I+]$ prion propagation [15]. PFAR has been described for the first time in vitro by the group of C. Das Gupta who showed that any denatured protein can recover its functional conformation thanks to domain $\mathrm{V}$ of $23 \mathrm{~S}, 25 \mathrm{~S}$ or $28 \mathrm{~S}$ rRNA [12]. Ribosome-assisted folding was then described for ribosomes from all living kingdoms and for all classes and protein sources tested $[12,23]$, which is consistent with the high conservation of the sequence and the secondary structure of rRNA domain $\mathrm{V}$ [13]. We previously identified anti-prion drugs 6AP and GA which also target PFAR in vitro [9] as well as in living bacteria [9] and yeast [15]. Although the main role of protein chaperones is to prevent protein misfolding and aggregation, the implication of a chaperone such as PFAR in propagating protein prion conformation is intuitive, since amyloid proteins exist in several conformations and their replication corresponds to the propagation of these particular conformations. This is illustrated by the role of Hsp104, a protozoan-specific protein chaperone that, together with Hsp70 and Hsp40, is the only cellular factor known to date to be essential for prion propagation $[P S I+]$ in yeast [52]. Using $[P S I+]$ as a prion model, we showed that, similarly to Hsp104, PFAR is involved in prion propagation [15]. Therefore, since PFAR fosters the propagation of pathologic protein conformation, inhibiting PFAR will allow ones to reduce the burden of aggregation of these pathologic proteins. Our present data confirm the therapeutic potential of anti-PFAR drugs and highlight this second enzymatic activity of the ribosome as an innovative therapeutic target for prion diseases.

OPMD is a dominant hereditary neuromuscular disease caused by the extension of a polyalanine repeat in the PABPN1 protein. Wild-type PABPN1 is localized in nuclear speckles, whereas polyalanine-expanded PABPN1 forms nuclear aggregates in skeletal muscle fibres, which is the pathological hallmark of OPMD. We thus evaluated the potential of anti-prion drugs on OPMD cellular and animal models. We previously showed that oral treatment with the anti-PFAR drugs 6AP and GA reduces OPMD-associated phenotypes, including defective wing posture due to thoracic muscle defects and the size of PABPN1 nuclear aggregates [23]. It should be noted that the OPMD Drosophila model previously allowed us to demonstrate a direct link between PFAR and the aggregation of mutated PABPN1 protein responsible for OPMD phenotypes [23]. Eight out of the ten new anti-PFAR drugs tested were also able to reduce the number of PABPN1 aggregates in a cell model of OPMD. Moreover, flunarizine, metixene and ebastine, which were more active than GA, were also able to alleviate muscle degeneration in the Drosophila OPMD model. Together, these data strengthen the role of PFAR in PABPN1 aggregation and highlight PFAR as a therapeutic target to treat OPMD. It is interesting to note that the activity spectrum of the drugs is remarkably similar for both diseases. Loperamide, which poorly crosses the blood-brain barrier, is a better candidate for OPMD than for prion diseases, but might be considered in prion diseases, to limit the peripheral propagation of $\operatorname{PrP}^{\mathrm{Sc}}$ in acquired forms of the diseases.

Flunarizine and some of its anti-prion structural analogs identified in this study have been previously assessed in other neurodegenerative disorders, some of which were also proteinopathies. Furthermore, flunarizine has recently been shown to increase impedance in learning and memory in a murine pharmacological model of Alzheimer's disease [53]. Flunarizine has also been shown to have a beneficial effect in spinal muscular atrophy (SMA) models [54], a hereditary neurodegenerative disease characterized by the death of spinal cord motor neurons and skeletal muscle atrophy. Survival motor neuron $(\mathrm{SMN})$ protein forms nuclear aggregates associated to Cajal bodies. Altered Cajal body localization of mutant SMN protein is a hallmark of SMA. Interestingly, flunarizine restores SMN localisation into the Cajal body of SMA-derived fibroblasts and spinal cord motor neurons in SMA mice, reduces the synaptic alterations of spinal cord motor neurons, improves motor function and expands survival of SMA mice [54]. Finally, flunarizine has also been shown to rescue the short lifespan of a C. elegans model of CLN3 disease (Spielmeyer-VogtSjogren-Batten disease), a paediatric-onset progressive neurodegenerative disease caused by recessive mutations in $C L N 3$, in which the $\mathrm{C}$ subunit of mitochondrial ATP synthase is known to abnormally accumulate and which is characterized by progressive vision loss, seizures, loss of cognitive and motor function 
and early death [55]. Loperamide has been shown to be able to clear A30P $\alpha$-synuclein aggregates in a Parkinson's disease cellular model [56], and reduce the accumulation of polyQ misfolded proteins in a Huntington's disease cellular model [57] by promoting autophagic degradation. We cannot exclude that the anti-prion activity of some drugs like loperamide, which is known to induce cell autophagy, could also be due to their capacity to activate autophagy.

Altogether, these data reinforce the concept that prion diseases share common mechanisms of pathogenic protein conformation propagation with other proteinopathies. They also suggest that PFAR may be a pertinent therapeutic target common to several of these diseases. It seems increasingly obvious that the success of the fight against these pathologies will come from complementary and synergistic therapies. Together, these data show that, despite being fundamentally different diseases, the common protein aggregation hallmark of protein misfolding diseases is a potent therapeutic target that could be used in combination with drugs targeting specific hallmarks of each disease. In this respect, anti-PFAR drugs like astemizole, azelastine, duloxetine, ebastine, flunarizine, loperamide and metixene appear to be of particular interest.

Supplementary Information The online version contains supplementary material available at https://doi.org/10.1007/s13311-020-00992-6.

Acknowledgments We thank G. Butler-Browne for the careful review of the manuscript; A. Nasir for the critical reading of the manuscript; Séverine Loisel, Marie-Françoise Scoazec and Manuel Feillant for the expert animal care; Hélène Simon and Johanna Mazé for their excellent technical assistance; Laetitia Guedeville, Laetitia Herzog, Fabienne Reine and Emilie Jaumain for the expert animal care and excellent technical assistance; and Fanny Roth for the technical assistance. This work was supported by Institut de France-Fondation NRJ (CV), MESR (AB, PHN), Inserm (CV and CT), UBO (CV), CNRS and University of Montpellier (MSg), AFM-Téléthon eOPMD project 17110 (CT and $\mathrm{MSg}$ ), Sorbonne Université and Association Institut de Myologie (CT), DIM MALINF, INRAE and Fondation pour la Recherche Médicale (Equipe FRM DEQ20150331689) (VB and SH), Association Défi Organisation $(\mathrm{CV})$. RNS held a PhD grant from AFM-Téléthon.

Required Author Forms Disclosure forms provided by the authors are available with the online version of this article.

\section{Compliance with Ethical Standards}

Disclaimer The funders had no role in study design, data collection and interpretation, or the decision to submit the work for publication.

Competing Interests he authors declare that they have no competing interests.

\section{References}

1. Prusiner SB. Novel proteinaceous infectious particles cause scrapie. Science 1982;216(4542):136-44. https://doi.org/10.1126/science. 6801762
2. Bach S, Talarek N, Andrieu T, et al. Isolation of drugs active against mammalian prions using a yeast-based screening assay. Nat Biotechnol 2003;21(9):1075-81.

3. Nguyen P, Oumata N, Soubigou F, et al. Evaluation of the antiprion activity of 6-aminophenanthridines and related heterocycles. Eur J Med Chem 2014;82:363-71. https://doi.org/10.1016/j.ejmech. 2014.05.083.

4. Nguyen PH, Hammoud H, Halliez S, et al. Structure-activity relationship study around guanabenz identifies two derivatives retaining antiprion activity but having lost alpha2-adrenergic receptor agonistic activity. ACS Chem Neurosci 2014;5(10):1075-82. https://doi.org/10.1021/cn5001588.

5. Oumata N, Nguyen PH, Beringue V, et al. The toll-like receptor agonist imiquimod is active against prions. PLoS One 2013;8(8): e72112. https://doi.org/10.1371/journal.pone.0072112.PONE-D13-23175.

6. Tribouillard-Tanvier D, Beringue $\mathrm{V}$, Desban $\mathrm{N}$, et al. Antihypertensive drug guanabenz is active in vivo against both yeast and mammalian prions. PLoS One 2008;3(4):e1981.

7. Voisset C, Saupe SJ, Galons H, Blondel M. Procedure for identification and characterization of drugs efficient against mammalian prion: from a yeast-based antiprion drug screening assay to in vivo mouse models. Infect Disord Drug Targets 2009;9(1):31-9.

8. Voisset C, Daskalogianni C, Contesse MA, et al. A yeast-based assay identifies drugs that interfere with immune evasion of the Epstein-Barr virus. Dis Model Mech 2014;7(4):435-44. https:// doi.org/10.1242/dmm.014308.

9. Voisset C, Thuret JY, Tribouillard-Tanvier D, Saupe SJ, Blondel M. Tools for the study of ribosome-borne protein folding activity. Biotechnol J 2008;3(8):1033-40. https://doi.org/10.1002/biot. 200800134.

10. Voisset C, Blondel M, Jones GW, et al. The double life of the ribosome: When its protein folding activity supports prion propagation. Prion 2017;11(2):89-97. https://doi.org/10.1080/19336896. 2017.1303587.

11. Chattopadhyay S, Pal S, Pal D, et al. Protein folding in Escherichia coli: role of $23 \mathrm{~S}$ ribosomal RNA. Biochim Biophys Acta 1999;1429(2):293-8.

12. Das D, Das A, Samanta D, et al. Role of the ribosome in protein folding. Biotechnol J 2008;3(8):999-1009. https://doi.org/10.1002/ biot.200800098.

13. Ben-Shem A, Garreau de Loubresse N, Melnikov S, et al. The structure of the eukaryotic ribosome at 3.0 A resolution. Science 2011;334(6062):1524-9. https://doi.org/10.1126/science.1212642.

14. Tribouillard-Tanvier D, Dos Reis S, Gug F, et al. Protein folding activity of ribosomal RNA is a selective target of two unrelated antiprion drugs. PLoS One 2008; 3(5):e2174.

15. Blondel M, Soubigou F, Evrard J, et al. Protein Folding Activity of the Ribosome is involved in Yeast Prion Propagation. Sci Rep 2016;6:32117. https://doi.org/10.1038/srep32117.

16. Scheckel C, Aguzzi A. Prions, prionoids and protein misfolding disorders. Nat Rev Genet 2018;19(7):405-18. https://doi.org/10. 1038/s41576-018-0011-4.

17. Gidaro T, Negroni E, Perie S, et al. Atrophy, fibrosis, and increased PAX7-positive cells in pharyngeal muscles of oculopharyngeal muscular dystrophy patients. J Neuropathol Exp Neurol 2013;72(3):234-43. https://doi.org/10.1097/NEN. $0 \mathrm{~b} 013 \mathrm{e} 3182854 \mathrm{c} 07$.

18. Tome FM, Fardeau M. Nuclear inclusions in oculopharyngeal dystrophy. Acta Neuropathol 1980;49(1):85-7. https://doi.org/10.1007/ bf00692226.

19. Brais B, Bouchard J-P, Xie Y-G, et al. Short GCG expansions in the $P A B P 2$ gene cause oculopharyngeal muscular dystrophy. Nat Genet 1998:18:164-7. 
20. Brais B, Bouchard JP, Xie YG, et al. Short GCG expansions in the PABP2 gene cause oculopharyngeal muscular dystrophy. Nat Genet 1998;18(2):164-7. https://doi.org/10.1038/ng0298-164.

21. Jouan L, Rocheford D, Szuto A, et al. An 18 alanine repeat in a severe form of oculopharyngeal muscular dystrophy. Can J Neurol Sci 2014;41(4):508-11. https://doi.org/10.1017/ s0317167100018588.

22. Richard P, Trollet C, Gidaro T, et al. PABPN1 (GCN)11 as a Dominant Allele in Oculopharyngeal Muscular Dystrophy Consequences in Clinical Diagnosis and Genetic Counselling. J Neuromuscul Dis 2015; 2(2):175-80. https://doi.org/10.3233/ JND-140060.

23. Barbezier N, Chartier A, Bidet $\mathrm{Y}$, et al. Antiprion drugs 6aminophenanthridine and guanabenz reduce PABPN1 toxicity and aggregation in oculopharyngeal muscular dystrophy. EMBO Mol Med 2011; 3(1):35-49. https://doi.org/10.1002/emmm. 201000109.

24. Malerba A, Roth F, Harish P, et al. Pharmacological modulation of the ER stress response ameliorates oculopharyngeal muscular dystrophy. Hum Mol Genet 2019. https://doi.org/10.1093/hmg/ ddz007.

25. Wermuth CG. Selective optimization of side activities: another way for drug discovery. J Med Chem 2004; 47(6):1303-14.

26. Wermuth CG. Multitargeted drugs: the end of the "one-targetone-disease" philosophy? Drug Discov Today 2004; 9(19): 826-7.

27. Kavitha CN, Kaur M, Jasinski JP, Yathirajan HS. Flunarizinium isonicotinate. Acta Crystallogr Sect E Struct Rep Online 2014; 70(Pt 6):o681-2. https://doi.org/10.1107/S1600536814010423.

28. Archer F, Bachelin C, Andreoletti O, et al. Cultured peripheral neuroglial cells are highly permissive to sheep prion infection. $\mathrm{J}$ Virol 2004; 78(1):482-90.

29. Feraudet C, Morel N, Simon S, et al. Screening of 145 anti-PrP monoclonal antibodies for their capacity to inhibit PrPSc replication in infected cells. J Biol Chem 2005; 280(12):11247-58. https://doi. org/10.1074/jbc.M407006200.

30. Vilotte JL, Soulier S, Essalmani R, et al. Markedly increased susceptibility to natural sheep scrapie of transgenic mice expressing ovine prp. J Virol 2001; 75(13):5977-84.

31. Le Dur A, Beringue V, Andreoletti O, et al. A newly identified type of scrapie agent can naturally infect sheep with resistant PrP genotypes. Proc Natl Acad Sci U S A 2005;102(44): 16031-6.0502296102. https://doi.org/10.1073/pnas. 0502296102 .

32. Langevin C, Andreoletti O, Le Dur A, Laude H, Beringue V. Marked influence of the route of infection on prion strain apparent phenotype in a scrapie transgenic mouse model. Neurobiol Dis 2011; 41(1):219-25. S0969-9961(10)00311-6. https://doi.org/10. 1016/j.nbd.2010.09.010.

33. Hasin N, Cusack SA, Ali SS, Fitzpatrick DA, Jones GW. Global transcript and phenotypic analysis of yeast cells expressing Ssa1, $\mathrm{Ssa} 2, \mathrm{Ssa} 3$ or Ssa4 as sole source of cytosolic Hsp70-Ssa chaperone activity. BMC Genomics 2014;15:194. https://doi.org/10.1186/ 1471-2164-15-194.

34. Parsell DA, Kowal AS, Lindquist S. Saccharomyces cerevisiae Hsp104 protein. Purification and characterization of ATP-induced structural changes. J Biol Chem 1994;269(6):4480-7.

35. Falsig J, Aguzzi A. The prion organotypic slice culture assayPOSCA. Nat Protoc 2008;3(4):555-62. https://doi.org/10.1038/ nprot.2008.13.

36. Raz V, Routledge S, Venema A, et al. Modeling oculopharyngeal muscular dystrophy in myotube cultures reveals reduced accumulation of soluble mutant PABPN1 protein. Am J Pathol 2011;179(4):1988-2000. https://doi.org/10.1016/j.ajpath.2011.06. 044.
37. Chartier A, Klein P, Pierson S, et al. Mitochondrial dysfunction reveals the role of mRNA poly(A) tail regulation in oculopharyngeal muscular dystrophy pathogenesis. PLoS Genet 2015;11(3):e1005092. https://doi.org/10.1371/journal.pgen. 1005092.

38. Amery WK. Brain hypoxia in migraine: pathophysiologic and therapeutic implications. J Cereb Blood Flow Metab 1982;2 Suppl 1: S62-5.

39. Amery WK. Flunarizine, a calcium channel blocker: a new prophylactic drug in migraine. Headache 1983;23(2):70-4.

40. Binnie CD, de Beukelaar F, Meijer JW, et al. Open dose-ranging trial of flunarizine as add-on therapy in epilepsy. Epilepsia 1985;26(5):424-8

41. Holmes B, Brogden RN, Heel RC, Speight TM, Avery GS. Flunarizine. A review of its pharmacodynamic and pharmacokinetic properties and therapeutic use. Drugs 1984;27(1):6-44. https:// doi.org/10.2165/00003495-198427010-00002.

42. Santi CM, Cayabyab FS, Sutton KG, et al. Differential inhibition of T-type calcium channels by neuroleptics. J Neurosci 2002;22(2): 396-403.

43. Karapetyan YE, Sferrazza GF, Zhou M, et al. Unique drug screening approach for prion diseases identifies tacrolimus and astemizole as antiprion agents. Proc Natl Acad Sci U S A 2013;110(17):70449. https://doi.org/10.1073/pnas.1303510110.

44. Kocisko DA, Baron GS, Rubenstein R, et al. New inhibitors of scrapie-associated prion protein formation in a library of 2000 drugs and natural products. J Virol 2003; 77(19):10288-94.

45. Srivastava KR, Lapidus LJ. Prion protein dynamics before aggregation. Proc Natl Acad Sci U S A 2017; 114(14):3572-7. https://doi. org/10.1073/pnas.1620400114.

46. Kocisko DA, Morrey JD, Race RE, Chen J, Caughey B. Evaluation of new cell culture inhibitors of protease-resistant prion protein against scrapie infection in mice. J Gen Virol 2004; 85(Pt 8): 2479-83. https://doi.org/10.1099/vir.0.80082-0.

47. Halliez S, Jaumain E, Huor A, et al. White blood cell-based detection of asymptomatic scrapie infection by ex vivo assays. PLoS One 2014;9(8):e104287. https://doi.org/10.1371/journal.pone.0104287.

48. Papamichael K, Delitheos B, Tiligada E. A subset of histamine receptor ligands improve thermotolerance of the yeast Saccharomyces cerevisiae. J Appl Microbiol 2013;114(2):492501. https://doi.org/10.1111/jam.12055.

49. Klein $\mathrm{P}$, Oloko $\mathrm{M}$, Roth $\mathrm{F}$, et al. Nuclear poly(A)-binding protein aggregates misplace a pre-mRNA outside of SC35 speckle causing its abnormal splicing. Nucleic Acids Res 2016;44(22):10929-45. https://doi.org/10.1093/nar/gkw703.

50. Chartier A, Benoit B, Simonelig M. A Drosophila model of oculopharyngeal muscular dystrophy reveals intrinsic toxicity of PABPN1. EMBO J 2006;25(10):2253-62.

51. Anvar SY, t'Hoen PA, Venema A, et al. Deregulation of the ubiquitin-proteasome system is the predominant molecular pathology in OPMD animal models and patients. Skelet Muscle 2011; 1(1):15. https://doi.org/10.1186/2044-5040-1-15.

52. Winkler J, Tyedmers J, Bukau B, Mogk A. Chaperone networks in protein disaggregation and prion propagation. $\mathrm{J}$ Struct Biol 2012;179(2):152-60. https://doi.org/10.1016/j.jsb. 2012.05.002.

53. Kamili C, Hemalatha E, Sowmya Kandoti H, et al. Evaluation of memory-enhancing effect of flunarizine on active avoidance in experimental model of Alzheimer's disease through calcium homeostasis. Int J Green Pharm 2019;13(4):348-53. https://doi.org/10. 22377/ijgp.v13i04.2706.

54. Sapaly D, Dos Santos M, Delers P, et al. Small-molecule flunarizine increases SMN protein in nuclear Cajal bodies and motor function in a mouse model of spinal muscular atrophy. Sci Rep 2018; 8(1):2075. https://doi.org/10.1038/s41598-018-20219-1. 
55. Kwon YJ, Falk MJ, Bennett MJ. Flunarizine rescues reduced lifespan in CLN3 triple knock-out Caenorhabditis elegans model of batten disease. J Inherit Metab Dis 2017;40(2):291-6. https://doi. org/10.1007/s10545-016-9986-1.

56. Williams A, Sarkar S, Cuddon P, et al. Novel targets for Huntington's disease in an mTOR-independent autophagy pathway. Nat Chem Biol 2008;4(5):295-305. https://doi.org/10.1038/ nchembio.79.
57. Zhang L, Yu J, Pan H, et al. Small molecule regulators of autophagy identified by an image-based high-throughput screen. Proc Natl Acad Sci U S A 2007;104(48):19023-8. https://doi.org/10.1073/ pnas.0709695104.

Publisher's Note Springer Nature remains neutral with regard to jurisdictional claims in published maps and institutional affiliations. 